risk of SARS-CoV-2 infection? Lancet Respir Med. 2020 Apr 3. Available from: https://www.sciencedirect.com/ sciencel article/pii/S2213260020301673?via\%3Dihub. Accessed April 30, 2020.

6. Brough HA, Kalayci O, Sediva A, Untersmayr E, Munblit $\mathrm{D}$, Rodriquez Del Rio $\mathrm{P}$, et al. Managing childhood allergies and immunodeficiencies during respiratory virus epidemics - the 2020 COVID-19 pandemic. Pediatr AllergyImmunol. 2020 Apr 22. Available from: https://onlinelibrary.wiley. com/doi/abs/10.1111/pai.13262. Accessed April 29, 2020.

7. COVID-19 Rapid Guideline: Severe Asthma. Available form:https://www.nice.org.uk/guidance/ng166/resources/ covid19-rapid-guideline-severe-asthma-pdf66141904108741. Accesed May 2, 2020.

8. Global Initiative for Asthma (GINA) 2020. Interim guidance on asthma management during the COVID-19 pandemic. Available from: https://ginasthma.org/ginareports/. Accessed April 29, 2020.

\section{COVID-19: Important Issues for Pediatricians}

Choudhary and Goyal [1] have raised important issues regarding issues affecting children during the SARSCoV-2 pandemic. We wish to highlight two additional issues related to the pandemic and the resultant lockdown.

A large number of children are likely to miss out on vaccinations due to postponement of campaigns and interruptions in routine vaccinations [2]. The Strategic Advisory Group of Experts (SAGE) on immunization recommended that all mass vaccination campaigns should be discontinued but routine immunization should continue where possible [3]. In keeping with this, the Indian government has issued guidelines advising continuation of routine immunization activities and the Indian Academy of Pediatrics - Advisory Committee on Vaccines and Immunization Practices (IAP-ACVIP) has issued guidelines for pediatricians in private practice $[4,5]$.

The immunization activities are mainly being carried out in fixed facilities with strict guidelines on hygiene and social distancing. Reduction in outreach immunization activities is likely to have an immediate impact on vaccine coverage. Health workers are involved in COVID-19 pandemic management, with decreased manpower available for routine immunization. The supply chain is also under strain due to transport disruptions. As immunization campaigns have been suspended, there will be a need for 'catch-up' campaigns, to identify those who missed their immunizations, as soon as the campaign is restarted.

The private sector is an important provider of immunization services in India. Reasons for the impact on immunization in private practice are lack of PPE for clinic staff, unavailability of vaccines and parent's inability to travel to clinic due to lockdown. Apart from the suggestions given by IAP-ACVIP [5], establishing a common 'community clinic' run by practicing pediatricians by rotation to offer vaccination and other services may also be explored. Moreover, electronic media and social media can be used to highlight the importance of continuing routine immunization services.

Another important issue is child abuse identification and prevention during the lockdown. During lockdown, children do not have access to any outside person to talk about the abuse that they face. They may be denied access to phone or any other mode of communication. Child Line services reportedly have a $50 \%$ increase in calls, many of them reporting child abuse [6]. The government has made efforts to ensure access to critical services such as healthcare, nutrition, food security, mental health and psychosocial support and protection against violence. Pediatricians need to be alert to the increased possibility of child abuse during the lockdown, and should report and liaison with the government authorities.

Funding: None; Competing interests: None stated. Published online: May 14, 2020; PII: S097475591600177

\section{Aarti A Kinikar* AND RaJesh K KulkarnI Department of Pediatrics, BJ Government Medical College, Pune, Maharashtra, *aarti.kinikar63@gmail.com \\ REFERENCES}

1. Choudhary B, Goyal JP. Management concern for noncovid children during the covid pandemic. Indian Pediatr. 2020 May 04. Available from: https://www.indian pediatrics.net/COVID29.03.2020/CORR-00173.pdf. Accessed May 06, 2020.

2. COVID-19: massive impact on lower-income countries threatens more disease outbreaks. Global Alliance for Vaccines and Immunisation Available from: https://www. gavi.org/news/media-room/covid-19-massive-impactlower-incomecountries-threatens-more-disease-outbreaks. Accessed April 19, 2020.

3. World Health Organization Guiding principles for immunization activities during the COVID-19 pandemic. 
Available from: https://www.who.int/immunization/policy/ sage/en/. Accessed April 19, 2020.

4. Ministry of Health and Family Welfare. Enabling Delivery of Essential Health Services during the COVID 19 Outbreak: Guidance note. Available from: https:// www.mohfw.gov.in/pdf/Essentialservicesduring COVID19updated0411201.pdf. Accessed April 19, 2020.
5. Indian Academy of Pediatrics. ACVIP Guidelines on Immunizations During COVID 19 Pandemic. Available from: https://iapindia.org/pdf/1455-FINAL-ADVISORYACVIP-Guidelines-on-Immunisations-during-COVID-19Pandemic-skd.pdf. Accessed May 06, 2020.

6. Child Line fielding calls against abuse. The Hindu.2020 April 08;New Delhi:p.6 (col3).

\section{Renal Complications in Children with Hematotoxic Snakebite: More Information Needed}

We read with interest the recent article on renal complications in children with hematotoxic snakebite by Islam, et al. [1]. We herein wish to raise some pertinent issues to assist in better understanding of this article.

(i) Though the aim of the study was to ascertain clinical and laboratory indicators predicting acute kidney injury (AKI) "early" in children with snakebite envenomation; these predictors have neither been mentioned in the results nor in tables, unlike an earlier study [2], where various clinical and laboratory parameters were reported as predictors.

(ii) There is no mention of baseline hemoglobin, maximum fall in hemoglobin, serum lactate dehydrogenase, evidence of myoglobinuria, hemodynamic status, cardiac dysrhythmias, cardiac dysfunction, evidence of adrenal hemorrhage, blood pressure, creatinine etc. which would have helped interpret the results better. These would have looked at creating a list of predictors of renal complications too [2]. Similarly, AKI could have been due to numerous other confounders like shock, dehydration, nephrotoxic antibiotics adminis-tration etc., which have not been detailed. Similarly, whether drug dose adjustments were made in those with AKI has also not been mentioned.

(iii) AKI was appropriately defined based on the Kidney Disease: Improving Global Outcomes (KDIGO) criteria [3]. However, these patients were then followed up for 6 months [1], the reason for which is not clear, because for labelling chronic kidney disease, a 3-month follow-up would have been enough.

(iv) Though one of the criteria for dialysis mentioned in this study was hyperkalemia, but the reason why medical management was not considered as an option is not apparent. Similarly, other reasons for dialysis like uremia, refractory metabolic acidosis too may have been indications for dialysis in these patients, which probably have not been included.

(v) It was mentioned in the methodology that "peritoneal dialysis was done in the institution and hemodialysis in a referral hospital". Whether these children were excluded or followed up is not clear. Details of how these children were followed up are missing. How many of these children who underwent dialysis developed 'permanent renal damage' at the 6-month follow up too has not been mentioned by authors, which could have been new information for the readers.

(vi) Similarly, it is not clear as to whether the authors had taken the AKI stage at presentation or the maximum AKI stage as per the KDIGO guidelines during the hospital stay.

(vii) What were the indications and timing for the renal biopsy? Was doing a renal biopsy in the setting of an AKI reasonably justified and ethically correct? Snake-bites being medicolegal cases, it looks improbable that a renal biopsy was possible in $100 \%$ of the children who died but in only $81.4 \%$ of those who survived.

(viii) It is mentioned that 59 out of 364 children (16.2\%) had "permanent renal damage" [1]. This is inappropriate as the denominator should exclude the deaths as permanent renal damage can be assessed only in those who survived the episode. So, we feel that the 16 children who succumbed should have been excluded, thus increasing the percentage of children with permanent renal damage to $16.9 \%$.

(ix) We presume that the median number of vials of antisnake venom (ASV) used in both groups have been mentioned in Table I [1]. It may have been appropriate to have also mentioned the mean value, which would have added more clarity to the renal outcomes. 